\title{
Turbulent Mass Transfer Optimization Control Technology in Coalmine
}

\author{
Shufang Wang ${ }^{*}, 1$, Zhiyong Yang $^{2}$, and Yeming Zheng ${ }^{1}$ \\ ${ }^{I}$ Department of Mechanical Engineering and Automation, Beijing Union University, Beijing 100020, China \\ ${ }^{2}$ Astronautics Long March rocket technology Limited company, Beijing City 100020, China
}

\begin{abstract}
Gas control in coalmine was the critical issue since gas accidents give rise to a lot of harm. This paper put forward the optimization control strategy based on the turbulent mass transfer mechanism on the basis of previous research. First of all, gas turbulent mass transfer mathematical model was established in heading laneway. Three aspects were describing in detail, gas diffusion law and quality entransy conservation when no wind, gas fluid flow process and momentum entransy conservation when no wind, ventilation airflow turbulent mass transfer process and mathematics when ventilation exits. Turbulent mass transfer optimization control strategy on the basis of the method of Lagrange multipliers was adopted. Moreover, turbulent mass transfer Euler equation which satisfy mass entransy dissipation extremum in turbulent mass transfer process. Finally, simulation experiments were carried out in the experimental platform; the data shows that turbulent mass transfer optimization control strategy was effective.
\end{abstract}

Keywords: Heading laneway, mass entransy dissipation extremum, optimization control, turbulent mass transfer,

\section{INTRODUCTION}

Gas disaster in coal mine had become an essential issue all over the world. According to statistics, most gas explosions occurred in the heading laneway. Gas drilling before excavation can effectively reduce the overall concentration of coal seam gas and coal and gas outburst phenomenon [1]. However, reference [2] showed that still a large number of gas outburst phenomena exited after drainage by drilling. As long-term equipment, auxiliary ventilation system was still a fundamental approach to reduce the possibility of gas explosions [3]. How to discharge gas into return laneway safety and quickly was still bottlenecks for coal production. Therefore, research the gas control strategy and technology in heading laneway became an important research topic.

Scholars in coalmine ventilation have researched on this problem for a long time. Reference [4] had explored gas migration regulation and control measurement in coal cutting field in detail. In this research, the numerical computation of gas migration processed in coal mine has been conducted using the k- $\varepsilon$ turbulent viscosity modeling method. Gas concentration distribution was obtained using computer simulation and experimentally verified using laboratory equipment. Based on this, reference [5] studied gas migration in gun dig heading laneway. Taking into account the displacement role of gas on the atmosphere, a gas convection-diffusion mathematical model had been established on the basis of mass conservation. In this model, semi-empirical method was adopted. It was necessary to offer air flow field in order to acquire the result of gas distribution. Heer den and Sullivan [6] simulated airflow and its dust distribution near the excavator using computational fluid dynamics. KW Moloney, IS Lowndes and others obtained distribution of air flow using assisted ventilation methods [7]. With technology innovation, automation excavator took over gun digging. Reference [8] showed that gas emission sources in excavator heading laneway include excavation head, rock and coal in conveyor and coal walls. With the increase of digging speed, gas emission increases at excavation head obviously. Moreover, gas emission increased from coal walls since coal walls reduce the average exposure time remarkably. Reference [9] analyzed the air flow structure of jet ventilation during excavating process, build a CFD models to research the gas behavior at roadway [10]. It offered the calculation method for average wind speed in excavation face space and return laneway.

Meanwhile, researchers paid more attention to explore the corresponding control strategy. Reference [11] designed an auxiliary ventilation device using converter. Therefore, some agreements had been reached that variable voltage variable frequency (VVVF) control is the accepted way to deal with this problem. In view of complex, uncertain and coupling characteristics of auxiliary ventilation system, it was difficult to build a perfect mathematical model to describe real situation of the heading laneways. With the development of control theory, intelligent control had been introduced into the gas discharging system. Fuzzy control theory has obtains great concern since it can simulate the characteristics of the human mind [12, 13]. Reference applied fuzzy control theory to deal with gas discharging system. It described a two-input single-output fuzzy control model for auxiliary fan [14]. Moreover, fuzzy control theory has also been used in ventilation for a long-distance tunnel as shown in reference [15]. Shufang Wang established conventional four input single output of dual mode fuzzy control model according to the characteristics of coal mine 
ventilation, calculated weights of the four inputs using AHP method and BP neural network method [16]. It was realized that mining ventilation physical mechanism of gas migration in heading laneway needs to dig further.

Based on previous studies, this paper described turbulent mass transfer physical model in heading laneway, turbulent mass transfer optimization control strategy and turbulent mass transfer Euler equation, and experiment results.

\section{MATHEMATICAL DESCRIBE OF THE TURBULENT MASS TRANSFER PROCESS IN HEADING LANEWAY}

For high gas mine, gas drainage was necessary before excavation. However, a lot of gas random effused when extraction. At the same time, gas continued effused at coal wall. For the gas discharge tunnel to the field staff to provide fresh air ventilation system at the same time set the local. The ventilation air flow got into the excavation heading through the air-duct; it mixed with gas and transferred along the heading laneway. This process was come down to turbulent mass transfer [12]. The physical model in heading laneway is shown in Fig. (1).

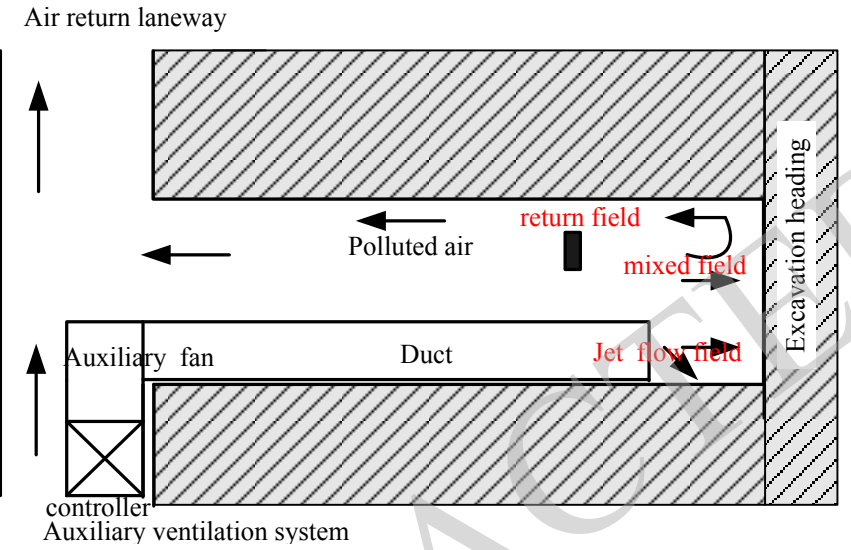

Fig. (1). Turbulent mass transfer physical model in heading laneway.

Turbulent mass transfer mathematical description needs the following assumptions. (1) Ventilation airflow can be regarded as a three-dimensional viscous incompressible fluid, ignoring the heat dissipation, and the fluid viscous isotropic, do scalar processing. (2) Assuming that the coal wall insulation, isothermal ventilation, seamless wind, and the wall without slipping. (3) Ventilation regarded as unsteady isothermal fluid. (4) Properties of wind and gas are constant, the mass transfer process in the absence of chemical reactions. (5) The air-duct outlet is the initial position, a given gas emission quantity is the initial conditions. In this paper, subscript a means air, g means gas. $Q_{\mathrm{g}}$ means emission quantity, $Q_{a}$ means ventilation air flow. Mass conservation, momentum conservation, energy conservation are satisfied for the whole heading laneway. That were $Q_{\text {in }}=Q_{\mathrm{g}}+Q_{a}=Q_{\text {out }} \quad, \quad P_{a}=m_{a} v_{a}+m_{g} v_{g}+\phi_{p}$ $W=W_{a}+W_{g}+\phi_{w}$, respectively. To facilitate the analysis, turbulent mass transfer in heading laneway was simplified. Firstly, mathematical description of gas diffusion and wind character was described; then adding ventilation airflow was added in model.

\subsection{Gas Diffusion Law and Mass Entransy Conservation When No Wind in Heading Laneway}

Gas concentration gradient induced diffusion movement when no ventilation airflow in the heading laneway. Gas diffusion motion which satisfies Fick diffusion law, $G_{\mathrm{g}}=-\rho D_{\mathrm{g}} \cdot \nabla Y_{\mathrm{g}} \cdot G_{\mathrm{g}}, \rho, D_{\mathrm{g}}, \nabla Y_{\mathrm{g}}$ was gas mass flux, gas density, gas diffusion coefficient, gas mass fraction gradient. $E_{v m}$ mass entransy was used to describe the ability of a certain component in the mixture of diffusion into the surrounding. Gas mass entransy $E_{v m g}=\frac{1}{2} G_{v m g} Y_{g}^{2}$. where, $G_{v m g}=\rho V Y_{g}$. Gas mass entransy in unit volume was $e_{v m g}=\frac{1}{2} \rho V Y_{g}^{2}$. Gas diffusion equation in heading laneway was rewritten as $\rho \frac{\partial Y_{g}}{\partial t}=-\nabla \cdot G_{g}$. The mass fraction $Y_{g}$ was multiplied at two sides of gas diffusion equation. Mass entransy balance equation was obtained as

$$
\rho Y_{g} \frac{\partial Y_{g}}{\partial t}=-\nabla \cdot\left(G_{g} Y_{g}\right)+G_{g} \nabla Y_{g}
$$

Mass entransy balance equation in unit volume was

$$
\phi_{v m g}=-\mathrm{G}_{g} \cdot \nabla Y_{g}=\rho D_{g}\left|\nabla Y_{g}\right|^{2}
$$

\subsection{Gas Fluid Flow Process and Momentum Entransy Conservation When Wind in Heading Laneway}

Firstly, the character of wind was described in detail in heading laneway. Reference [9] offered wind character. In fact, gas concentration distribution in heading laneway was associated with gas emission speed and volume at different gas resources, length of a heading laneway and air flow character. Air flow characters should be considered remarkably.

Since air was shot into heading laneway by auxiliary fan through round duct along coal wall, air flow was a kind of restrained wall attached jet flows. In general, duct was placed at side wall in heading laneway. The characteristics of it are different from free jet flow subjected to the limitations of laneway wall. It should be defined as round restrained wall attached jet flow. Through the duct outlet, air flow followed the freedom jets law of development at first. Due to the limited space in heading laneway, some air flows in the opposite direction then. As a result, air flow structure could be divided into a jet flow field, a mixed field and a return flow field. In the jet field, part of the air flow comes from the duct outlet and the other part comes from the rolled return air. Meanwhile, air flow of the return field was rolled by the jet flow, and others are discharged along the laneway. The form of the round restrained wall attached jet flow was shown in Fig. (2).

In heading laneway, gas migration process owned special character as shown in reference [5]. Mixing and discharging 


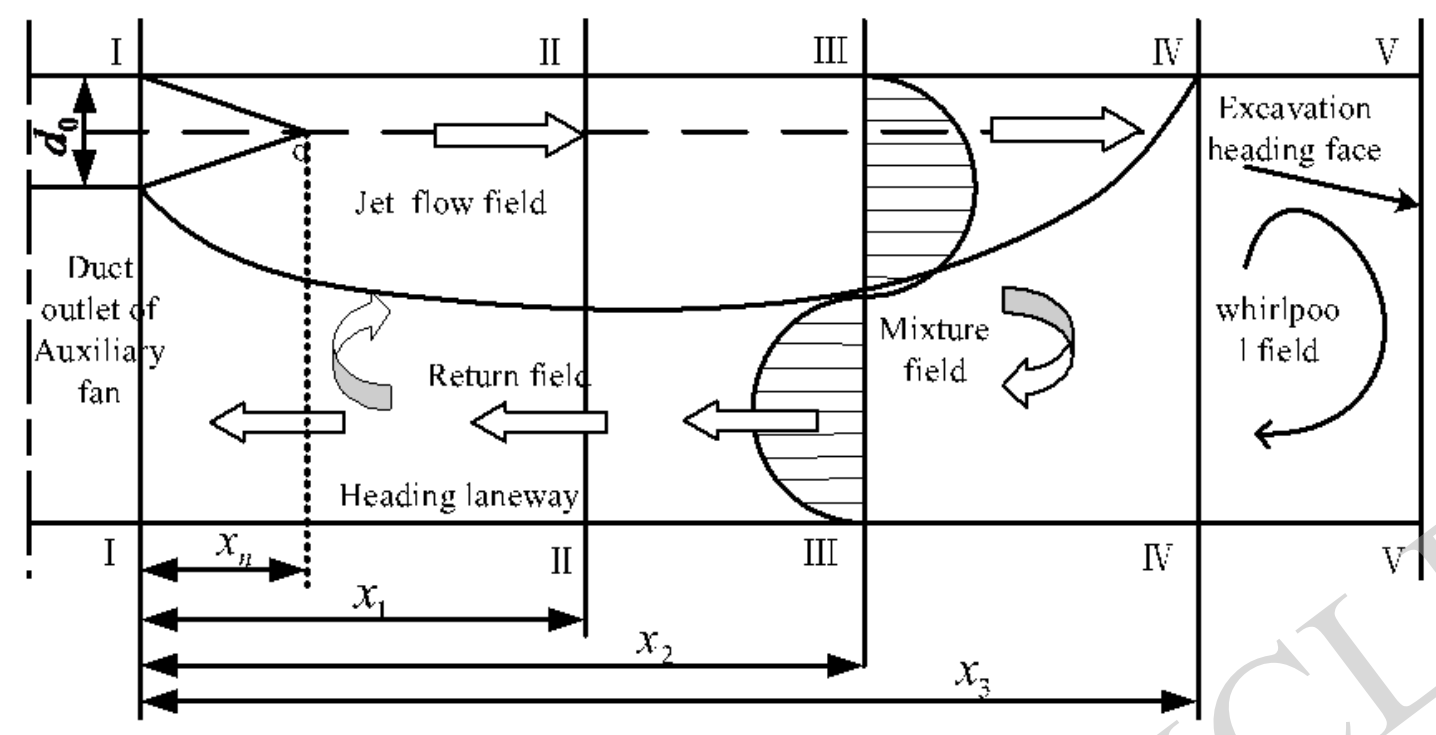

Fig. (2). Form of round restrained wall attached jet flow.

were main operations during gas migration process. Distribution of gas in heading laneway was described as gas concentration gradient. According to the law of molecular diffusion, gas diffuses from regions with high concentration to those with low concentration until it was uniformly distributed based on Fick's first law of diffusion, $J_{M}=-D_{m} \cdot \nabla c$, where $J_{M}$ was the molecular diffusion flux, $D_{m}$ was the molecular diffusion coefficient and $c$ is the gas concentration.

In order to express it easily, an infinitesimal cube was chose. In the cube, dimension $d x, d y$, and $d z$ with center $P(x, y, z)$ at in heading laneway was considered.

Moreover, the gas convection - diffusion equation could be formulated as

$\frac{\partial c}{\partial t}=\frac{\partial}{\partial x i}\left(D i j \frac{\partial c}{\partial x j}+D M \frac{\partial c}{\partial x i}\right)-(V x i+V p x i) \frac{\partial c}{\partial x i}+f$ where, $x i(x j)=\mathrm{x}, \mathrm{y}$, or $\mathrm{z}, c$ is the average gas concentration at volume center $P(x, y, z)$ with unit $g / m^{3}, V x i$ was the average wind speed in $m / s$, Vpxi was the displacement velocity component in each axis in $\mathrm{m} / \mathrm{s}, D_{m}$ was the molecular diffusion coefficient in $\mathrm{m}^{2} / \mathrm{s}$ and $D i j$ was the nine components of gas diffusion coefficient, $f$ was air flow field. Gas concentration was known at time $t=0$. Given gas concentration on the boundary $S 1$ was $\left.c(x, y, z, t)\right|_{(x, y, z) \in S 1}=c 1(x, y, z, t), c 1(x, y, z, t)$ was a function which can be calculated. At infinity distance away from the gas emission source, gas concentration was generally assumed to be zero. The gas concentration at the boundary between gas and air flow was given by higher gas concentration.

Then the process of gas diffusion was also momentum transfer. The fluid flow rate $u$ was strength variable, fluid momentum $P=\rho V u$ was extensive variable. Momentum

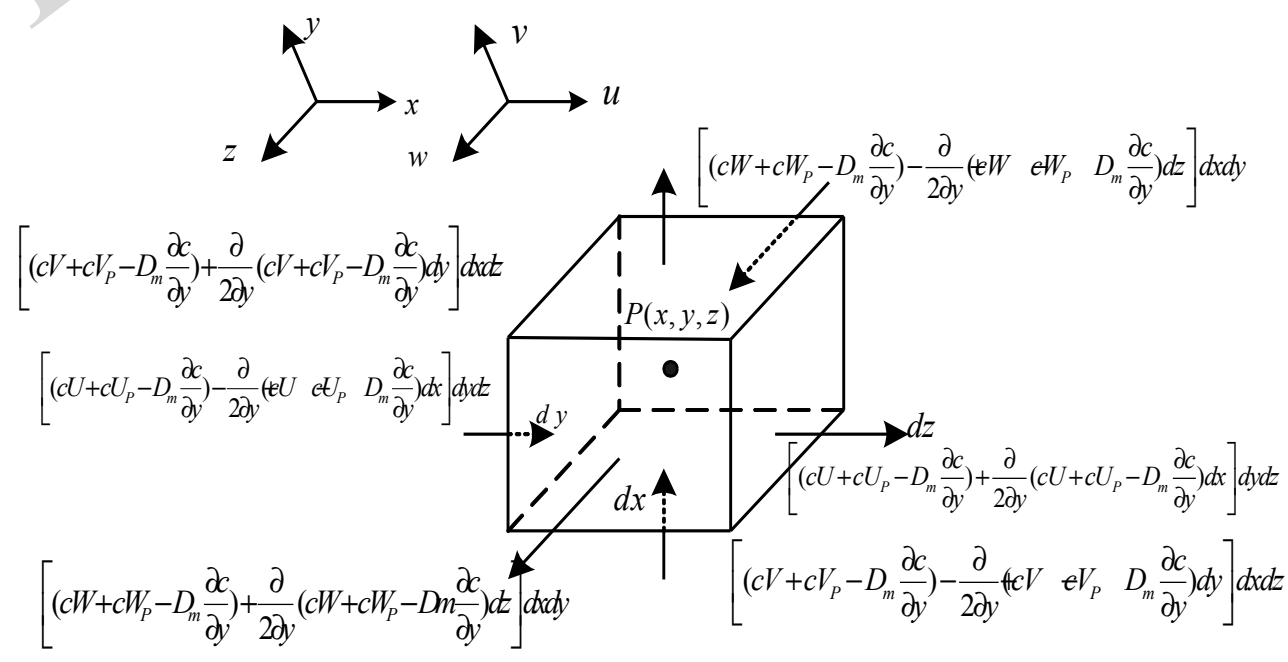

Fig. (3). Infinitesimal of heading interface wall at attached jet ventilation. 
entransy $E_{\text {mom }}$ was used to describe the fluid momentum diffusion ability. Gas momentum entransy $E_{\text {momg }}=\frac{1}{2} Q_{\text {momg }} u=\frac{1}{2} \rho V u^{2}$, momentum entransy in unit volume $e_{\text {momg }}=\frac{1}{2} \rho u^{2}$. The momentum equation of gas fluid $\frac{d P}{d x}=\mu \frac{d^{2} u}{d y^{2}}$. Where, $\mu$ was gas fluid viscosity coefficient.

The $u$ was multiplied at two sides of gas momentum equation. Momentum entransy balance equation was obtained as

$u \frac{d P}{d x}=\mu \frac{d\left(u \frac{d u}{d y}\right)}{d y}-\mu\left(\frac{d u}{d y}\right)$

Momentum entransy balance equation in unit volume was

$\frac{d P}{d x}=-\frac{d \dot{\mathrm{e}}_{m o n g}}{d y}-\phi_{m o n g}$

Ventilation airflow turbulent mass transfer process and mathematics when wind exits in heading laneway.

According to Boussinesq hypothesis, when gas turbulent mass transfer by ventilation in heading laneway, the gas mass conservation equation $\rho U \cdot \nabla Y_{g}=\nabla\left(\rho D_{g} \nabla Y_{g}\right)$. Where, $U$ was turbulent velocity vector. The mass fraction $Y_{g}$ was multiplied at two sides of gas mass conservation equation, $\rho U \cdot \nabla\left(\frac{Y_{g}^{2}}{2}\right)=\nabla\left(\rho D_{g} Y_{g} \nabla Y_{g}\right)-\rho D_{g}\left|\nabla Y_{g}\right|^{2}$. The left side of the equation expressed gas mass entransy transfer with motion of fluid particles, the first item at right side expressed gas mass entransy diffusion in fluid, the second expressed gas mass entransy dissipation, which is reversible process measure of the convective mass transfer.

Divided the control volume boundaries into mass transfer surface, the vast surface, the fluid inlet and the fluid outlet, mass transfer equilibrium equation can be expressed as

$$
\begin{aligned}
& \int_{\Gamma} \vec{n} \bullet\left(\rho D_{g} \nabla Y_{g}\right) d S=\int_{m s} \vec{n} \bullet\left(\rho D_{g} \nabla Y_{g}\right) d S+\int_{n m s} \vec{n} \bullet\left(\rho D_{g} \nabla Y_{g}\right) d S \\
& +\int_{\text {in }} \vec{n} \cdot\left(\rho D_{g} \nabla Y_{g}\right) d S+\int_{\text {out }} \vec{n} \bullet\left(\rho D_{g} \nabla Y_{g}\right) d S
\end{aligned}
$$

Due to the viscous effect, gas mass transport and fluid flow need to consume the mechanical energy in the process of turbulent mass transfer in heading laneway. Thus the optimization work can be taken as two conditions. One is mass entransy dissipation extremum at given momentum entransy dissipation, the other momentum entransy dissipation extremum at given mass entransy dissipation. For turbulent mass transfer in heading laneway, in order to find flow field that meet mass dissipation minimum at momentum entransy dissipation condition. It is required research air flow field which mass entransy dissipation was extremum at given pump power. That means viscous dissipation extremum at given mass entransy dissipation in the process turbulent mass transfer. For the sake of realizing the optimization velocity field at given constraint conditions the, the mathematical model was depicted for the extremum constrained functional.

\section{OPTIMAL CONTROL STRATEGY BASED ON LAGRANGE MULTIPLIER METHOD OF ROADWAY TURBULENT MASS TRANSFER}

Lagrange multipliers method transformed solve constrained functional extremum into unconstrained. Moreover, variational method is adopted to solve unconstrained functional extreme. Turbulent mass transfer mathematical description as optimization object, optimization objectives, constraints and boundary condition. The optimization object was velocity field, optimization aims was mass entransy $\phi_{\text {mong }}=\rho D_{g}\left|\nabla Y_{g}\right|^{2}$ extremum, the constraint condition was given momentum entransy dissipation, $\phi_{\text {mong }}=\mu\left(\frac{\partial u_{j}}{\partial x_{i}}+\frac{\partial u_{i}}{\partial x_{j}}\right) \frac{\partial u_{i}}{\partial x_{j}}$, boundary conditions for the continuity equation $\phi_{m o n g}=\nabla \cdot(\rho U)=0$ and gas component mass conservation equation $\rho \mathrm{U} \cdot \nabla Y_{g}=\nabla\left(\rho D_{g} \nabla Y_{g}\right)$.

Lagrange function is constructed under the given conditions

$$
\Pi=\iiint_{\Omega}\left[\phi_{v m g}+C_{0} \phi_{m o n g}+A\left(\rho D_{g} \cdot \nabla Y_{g}-\rho U \cdot \nabla Y_{g}\right)+B \nabla \cdot \rho U\right] d V
$$

where, $A, B$ was space position function, $C_{0}$ was constant. Equation (6) variation method was used to deal with mass fraction $Y_{g}$, velocity vector, $A, B$ respectively,

$$
\begin{aligned}
& -\rho \mathrm{U} \cdot \nabla A=\rho D_{g} \nabla \cdot(\nabla A)-2 \rho D \cdot\left(\nabla Y_{g}\right) \\
& \mu \nabla^{2} \mathrm{U}+\frac{\rho}{2 C_{0}} A \nabla Y_{g} D_{g}+\frac{1}{2 C_{0}} \nabla B=0 \\
& \rho U \cdot \nabla Y_{g}=\nabla\left(\rho D_{g} \nabla Y_{g}\right) \\
& \nabla \cdot(\rho U)=0
\end{aligned}
$$

The simultaneous solution of the above equation can be drawn on the distribution of unknown variables in the turbulent mass transfer region. Meanwhile, Gas fluid must satisfy the momentum equation

$$
\rho U \cdot \nabla U=-\nabla P+\mu \nabla^{2} \mathrm{U}+F
$$

Comparing (8) and (11),

$\rho U \cdot \nabla U+\nabla P-F=\mu \nabla^{2} \mathrm{U}=-\frac{\rho}{2 C_{0}} A \nabla Y_{g}-\frac{1}{2 C_{0}} \nabla B$

$B=-2 C_{0} P$, 


$$
F=\rho U \cdot \nabla U+\frac{\rho}{2 C_{0}} A \nabla Y_{g}=\rho U \cdot \nabla U+C_{\Phi} A \nabla Y
$$

where, $F$ was the virtual additional volume force. The mass entransy dissipation extremum Euler equation can be obtained by putting $\mathrm{F}$ expression into momentum equation.

$$
\begin{aligned}
& \rho U \cdot \nabla U=-\nabla P+\mu \nabla^{2} U+F \\
& =-\nabla P+\mu \nabla^{2} U+\rho U \cdot \nabla U+C_{\Phi} A
\end{aligned}
$$

\section{EXPERIMENT PLATFORM}

Turbulent mass transfer experiment platform includes control systems, heading laneway model, gas source simulation, speed adjustment and signal detection. 1-8 represents air-duct, partial fan, excavation gas emission duct, coal wall gas emission duct, excavation heading, heading laneway, control system and converter, respectively. As shown in Fig. (4). Acceptable simplification is adopted since it was difficult to simulate heading laneway environment in coal mine. For example, coal wall roughness of heading laneway simulation model was ignored; the normal room temperature and humidity was adopted instead of underground coal mine. Nevertheless, the experiment system still simulation the correlation which existed between the value of wind speed sensor and the value of wind speed at duct outlet. Moreover, PLC controller completes the dualmode fuzzy control strategy based on weighting coefficients.

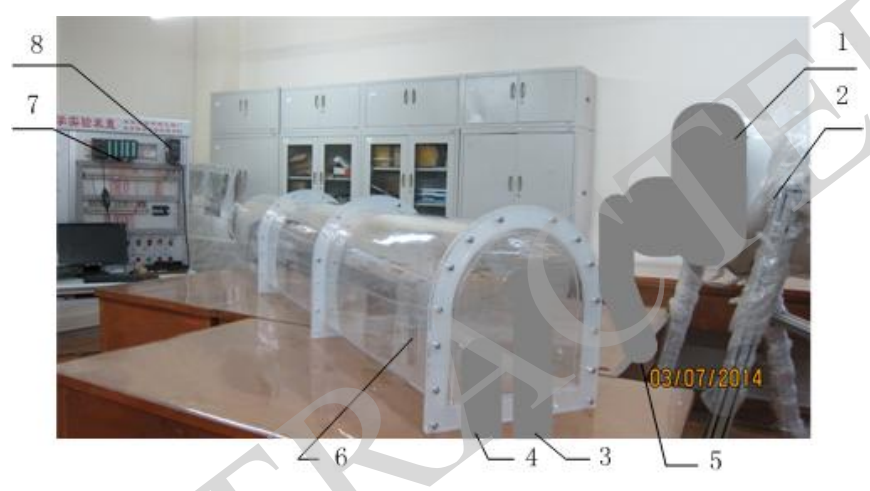

Fig. (4). Turbulent mass transfer platform in heading laneway.
According simulation results with Anysis software, the main wind characteristics and gas concentration gradient changing occur within 10 meters from air-duct outlets to excavation heading. Wind characteristics emitted from airduct including jet zone, mixing zone, recirculation zone. After recirculation zone, gas concentration and wind speed had been basically uniform and stable. Therefore, the sensor arrangement of the experiment was mainly considered in the case of $10 \mathrm{~m}$. Signal sensors include 21 micro-gas concentration sensors G1-G21 and 16 wind speed sensors $\mathrm{W} 1-\mathrm{W} 16$. They are arranged at a distance of $0.1,0.2 \mathrm{~m} 0.4$ $\mathrm{m}, 0.8 \mathrm{~m}, 1.0 \mathrm{~m}$ was arranged on the cross section of the same with $0.1 \mathrm{~m}$. as shown in Fig. (5).

Due to the volume of gas was less than the density of the air, the gas concentration was significantly higher than other locations on the top of the heading laneway. The results before and after optimization were shown that gas concentration distribution was more uniform by adding additional volume force. It indicated that gas transfer rate is increasing after optimization.

\section{EXPERIMENT RESULTS AND DISCUSSIONS}

One set of comparative experimental results were discussed only. After adjusting the gas pump valve so that G1 detected concentration of $0.8 \%$, partial fan was started up at $30 \mathrm{~Hz}$ frequency. The velocity of air-duct outlet was 2 $\mathrm{m} / \mathrm{s}$. Experiment data before optimization and after

\begin{tabular}{|c|c|c|c|c|c|c|c|}
\hline Section & \multicolumn{4}{|c|}{ Gas (\%) } & \multicolumn{3}{|c|}{ Wind $(\mathrm{m} / \mathrm{s})$} \\
\hline $0.1 \mathrm{~m}$ & 0.8 & 0.82 & 0.75 & 0.61 & 1.41 & 1.45 & 1.52 \\
\hline $0.2 \mathrm{~m}$ & 0.75 & 0.80 & 0.65 & 0.52 & 1.29 & 1.33 & 1.68 \\
\hline $0.4 \mathrm{~m}$ & 0.71 & 0.78 & 0.61 & 0.48 & 1.24 & 1.12 & 1.79 \\
\hline $0.8 \mathrm{~m}$ & 0.54 & 0.65 & 0.49 & 0.41 & 1.13 & 0.84 & 1.91 \\
\hline $1.0 \mathrm{~m}$ & 0.46 & 0.52 & 0.45 & 0.31 & 0.95 & 0.75 & 2.0 \\
\hline $3.0 \mathrm{~m}$ & \multicolumn{4}{|c|}{0.35} & \multicolumn{3}{|c|}{0.90} \\
\hline
\end{tabular}
optimization were shown in Tables $\mathbf{1}$ and $\mathbf{2}$.

Table 1. Experiment data before optimization.

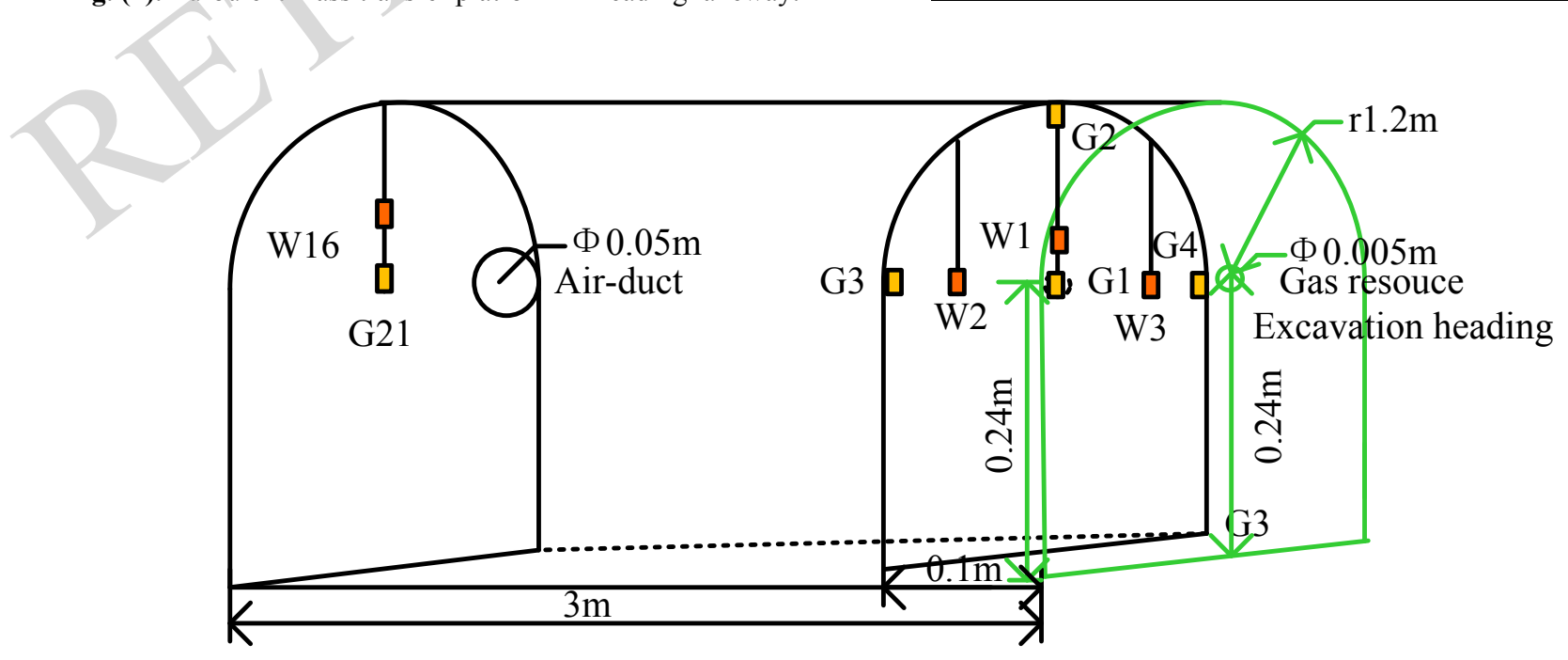

Fig. (5). Schematic diagram of sensor placement. 
Table 2. Experiment data after optimization.

\begin{tabular}{|c|c|c|c|c|c|c|c|}
\hline Section & \multicolumn{4}{|c|}{ Gas (\%) } & \multicolumn{3}{c|}{ Wind (m/s) } \\
\hline \hline $0.1 \mathrm{~m}$ & 0.8 & 0.82 & 0.75 & 0.61 & 1.41 & 1.45 & 1.52 \\
\hline $0.2 \mathrm{~m}$ & 0.72 & 0.79 & 0.65 & 0.52 & 1.29 & 1.33 & 1.68 \\
\hline $0.4 \mathrm{~m}$ & 0.69 & 0.74 & 0.61 & 0.48 & 1.24 & 1.12 & 1.79 \\
\hline $0.8 \mathrm{~m}$ & 0.56 & 0.63 & 0.49 & 0.41 & 1.13 & 0.84 & 1.91 \\
\hline $1.0 \mathrm{~m}$ & 0.48 & 0.50 & 0.45 & 0.31 & 0.95 & 0.75 & 2.0 \\
\hline $3.0 \mathrm{~m}$ & \multicolumn{3}{|c|}{0.38} & \multicolumn{5}{c|}{0.94} \\
\hline
\end{tabular}

\section{CONCLUSION}

This paper described the establishment process of turbulent mass transfer optimal control strategy in heading laneway in coal mine. Control strategies optimization requirement was put forward to meet the needs of different gas concentrations at different regions in heading laneway. The turbulent mass transfer mathematical was depicted in detail. Gas diffusion law and quality entransy conservation when no wind, gas fluid flow process and momentum entransy conservation when no wind, ventilation airflow turbulent mass transfer process and mathematics when ventilation exited. Specifically, gas control optimization strategies was aim to obtain mass entransy dissipation extremum at given turbulent mass transfer entransy. Then velocity field was optimized at given constraint conditions. Therefore, the problem was attributed to a constrained functional extremum math problem. The solving method was transformed into unconstrained optimization functional by using the method of Lagrange multipliers. Then variational method was adopted to solve functional. Finally, the additional volume force that optimal control strategies required was obtained. The experimental results verify the turbulent mass transfer platform, optimize control strategy had certain effect in the rational distribution of air flow field.

\section{CONFLICT OF INTEREST}

The author confirms that this article content has no conflict of interest.

\section{ACKNOWLEDGEMENTS}

This paper is funded by National Nature Science Foundation of China 41102098, The Importation and
Development of High-Caliber Talents Project of Beijing Municipal Institutions.

\section{REFERENCES}

[1] C. O. Karacan, W. P. Diamond, and S. J. Schatzel, "Numerical analysis of the influence of in-seam horizontal methane drainage boreholes on longwall face emission rates", International Journal of Coal Geology, vol. 72, pp. 15-32, 2007.

[2] X. J. Li, "Theory and practices on high level borehole gas drainage technology", coal science and technology, vol. 4, pp. 16-18, 2007.

[3] K. Wang, and Q. X. Yu, "Regular rule and controlling means of gas emission at coal face in China", Northwest coal, vol. 2, pp. 3-7, 2007.

[4] D. Liang, Ventilation process gas migration patterns and numerical simulation, Beijing: Coal Industry Press, 1999.

[5] E. Y. Wang, Alone in the first tunnel airflow characteristics of gas transport, Fuxin: Fuxin Mining College, 1994

[6] J. L. T Azevedo, and J. C. F. Pereira, "Modeling of multisized particle laden turbulent low swirling free jet", Journal of Wind Engineering and Industrial Aerodynamics, vol. 46-47, pp. 713-720, 1994.

[7] S. Nakayama, K. Uchino, and M. Inoue, "Analysis of ventilation air flow at heading face by computational fluid dynamics", Shigen To - Sozai, vol. 111, no. 4, pp. 225-230, 1995.

[8] F. Qu, "Methane Emission Rate Prediction in Fully-mechanized Excavated Faces Based on Initial Speed of Methane Emission of Coal Wall", Safety in Coal Mines, vol. 8, pp. 1-4, 2004.

[9] H. Q. Wan, and S. L. Shi, "Numerical simulation study on ventilation flow field of wall-attached jet in heading face", Journal of China Coal Society, vol. 29, pp. 425-428, 2004.

[10] J. Torano, S. Torno, and M. Menendez, "Models of methane behaviour in auxiliary ventilation of underground coal mining", Tunnelling and Underground Space Technology, vol. 80, pp. 3543, 2011.

[11] J. F. Li, "The development of partial ventilation safety and energy saving monitoring devices", Coal Mine Machinery, vol. 4, pp. 13$17,2003$.

[12] L. J. Zhu, and R. L. Wang, "Fuzzy control technology for mine local fan with frequency Inverter speed control", Coal Science and Technology, vol. 4, pp. 7-9, 2003.

[13] E. Karakas, "The control of highway tunnel ventilation using fuzzy logic", Engineering Applications of Artificial Intelligence, vol. 16, pp. 717-721, 2003.

[14] M. H. Hassoun, Fundamentals of Artificial Neural Networks, Massachusetts: MIT Press, 1995.

[15] R. Y. Chen, and S. F. Wang, "Analytical Hierarchy Process and ANN Applied in Gas Discharging Fuzzy Model," in Proceedings of The $8^{\text {th }}$ World Congress on Intelligent Control and Automation, July 7-9, 2010, Jinan, China, pp. 4992-4995.

[16] S. F. Wang, D. B. Zhou, and Z. Y. Yang, "Fuzzy Control Technique of Auxiliary Ventilation in Heading Laneway", Research Journal of Applied Science, Engineering and Technique, vol. 5, no. 3, pp. 914-921, 2013. 